慶應義塾大学学術情報リポジトリ

Keio Associated Repository of Academic resouces

\begin{tabular}{|c|l|}
\hline Title & Problem of large deflection of coiled springs \\
\hline Sub Title & \\
\hline Author & 水野, 正夫(Mizuno, Masao) \\
\hline Publisher & 慶応義塾大学藤原記念工学部 \\
\hline Publication year & 1959 \\
\hline Jtitle & $\begin{array}{l}\text { Proceedings of the Fujihara Memorial Faculty of Engineering Keio } \\
\text { University Vol.12, No.44 (1959.),p.18(18)- 35(35) }\end{array}$ \\
\hline JaLC DOI & \\
\hline Abstract & $\begin{array}{l}\text { The deflection curve of the center line of coiled springs are discussed, expanding the Bernoulli- } \\
\text { Euler equation for the elastica of thin rods. } \\
\text { Numerical results and their application to the springs for measuring pressure of dial gauges are } \\
\text { presented. }\end{array}$ \\
\hline Notes & The twentieth anniversary memorial volume \\
\hline Genre & Departmental Bulletin Paper \\
\hline URL & $\begin{array}{l}\text { https://koara.lib.keio.ac.jp/xoonips/modules/xoonips/detail.php?koara_id=KO50001004-0012004 } \\
\text { 4-0018 }\end{array}$ \\
\hline
\end{tabular}

慶應義塾大学学術情報リポジトリ(KOARA)に掲載されているコンテンツの著作権は、それぞれの著作者、学会または出版社/発行者に帰属し、その権利は著作権法によって 保護されています。引用にあたっては、著作権法を遵守してご利用ください。

The copyrights of content available on the KeiO Associated Repository of Academic resources (KOARA) belong to the respective authors, academic societies, or publishers/issuers, and these rights are protected by the Japanese Copyright Act. When quoting the content, please follow the Japanese copyright act. 


\title{
Problem of Large Deflection of Coiled Springs**
}

\author{
(Received Oct. 10, 1959)
}

Masao MIZUNO*

\begin{abstract}
The deflection curve of the center line of coiled springs are discussed, expanding the Bernoulli-Euler equation for the elastica of thin rods.

Numerical results and their application to the springs for measuring pressure of dial gauges are presented.
\end{abstract}

\section{Introduction}

When the elastic deformation in bending of bars, plates etc. is discussed from the view-point of the strength of materials and applied elasticity, it is a customary procedure to treat it under the following fundamental assumptions.

(1) The equilibrium of stresses between themselves and with external forces needs only to hold under the form and conditions of the undeformed states.

(2) The squares of deformation angles are negligible compared to unit. As these fundamental assumptions are only approximately valid in small deformations, the theory grounded on them is called the theory of infinitesimal deformations. In this theory all the strains and stresses caused by external forces are linear in them, and uniqueness of the deformed state corresponding to definite external forces is demonstrated and a stable equilibrium will be established. Therefore (1) of the fundamental assumptions must be regarded as not to valid in the theory of elastic stability of bars, such as the one of buckling of columns. Such a theory is called one of finite deformations. In larger deformations, it will be necessary to treat (2) of the fundamental assumptions as not true. Then we have problems of

*水野正夫 Dr. Eng., Assistant Professor at Keio University.

** Contents of this paper were read at the following meetings under the title "Deformation of Coiled Springs under Oblique Loads (Part 1 4.)":

Part 1: General meeting to the Japan Society of Mechanical Engineers, April 1955 (Lecture No. 132).

Part 2: Extraordinary general meeting of the Japan Society of Mechanical Engineers, November, 1956 (No. 119).

Part 3: The 60th anniversary meeting of the Japan Society of Mechanical Engineers in the second region, July, 1957 (No. 109).

Part 4: Autumnal meeting of the Society for Precision Mechanics of Japan, November, 1957 (No. 204). 
large deflection. The large deflection problem of bars of uniform section is called the problem of elastica and was discussed by D. Bernoulli and L. Euler for the first time, so we must regard it as a classical problem. ${ }^{1)}$ But the theory of infinitesimal deformations is simple and the test results are pretty close to the real deformations and it is often taken as a sufficient approxmation for practial use except for exceptionally large deformations. So, as a practical computation method, it has been used until now more widely thae the problem of elastica. Majority of the ordinary elastic theories such as those of elasticity, strength of materials, structures etc. belong to this theory of infinitesimal deformations. ${ }^{2)}$ A number of papers published recently concerning the large deflection problem is much less than the number of those founded on the theory of infinitesimal deformations. We can only name the work of Suhara, discussing the elastica of bars of gradually varied section, ${ }^{1)}$ the work of E. J. Scott and others, the solution in series being obtained by reversion method, ${ }^{3)}$ the work of H. D. Conway etc, discussing the large deflection of bars curved in circular arc," the work of Hamada, treating the large deflection of plate ${ }^{5>}$ and a few others, all of them treating bending deflection. Generally speading, a spring is a machine element characterised by a large deformation in the whole even when its elements may be regarded to have infinitesimal strains in the elastic range. Therefore, in order to get an accurate result, the computation of its strains and stresses must be carried out as problem of large deflection, but this is scarcely ever porformed. There is only one work by Watari on laminated springs. ${ }^{6}$ ) The author treats in this paper the deflection of a coiled spring in the state of very large deformations. Theoretically sound resnlts are obtained only by the treatment of large deflection problem. However, such treatment will scarcely find a practical importance except in case of these springs. ${ }^{7)}$

\section{Fundamental Equations for the Problem of Large Deflection of Coiled Springs}

In ordes to determine the deflection curve of the central line of a buckled coiled spring, we treat the central line as a bar with a certain rigidity, then the spring. will be subjected to a direct force depending on the position of each point on its

1) Suhara: Journal of the Japan Society of Mechanical Engineers, 41, No. 255, 583 (1938, June) [in Jap.]

2) Risearch committee of long columns: Hand book of Elastic Stability, p. 2, Corona Book Co. (1951).

3) E. J. Scott and D. R. Carver: J. App. Mech. June 1955, p. 245.

4) H. D. Conway: J. App. Mech. March 1956, p. 7.

5) Hamada : Bulletin of JSME vol. 1, No. 1 (1958) 20. [in Eng.]

6) Watari: Preprint for the Tokyo meeting of the Japan Society of Mechanical Engineers (Oct. 1955), No. 109,

7) cf. The resume (1). 
central line, and so its pitch varies from point ot point, i.e. we get a case of a bar with gradually varying rigidity. The elastica of a bar with gradually varying cross section has been treated by Suhara ${ }^{8)}$ and myself ${ }^{9}$, but the spring in the present case is more complicated. We must take into consideration the curvature of its central line caused by a varying shear which is comparable with the curvature caused by bending and cannot be neglected and also the gradually varying rigidity which is brought about by the dependence of the total length on its load. We assume that the coiled spring with uniform pitches and straight central line in its undeformed state is buckled under pressure $R$ acting on both hinged ends, and the deformed curve of the central line is $A P O B$, as shown in Fig. 1. The deformed curve has a parallel tangent to the force $R$ in its central point $O$. As in Fig. 1, let this tangent be ordinate and $O$ be the origin. Then the deformed curve will be symmet-

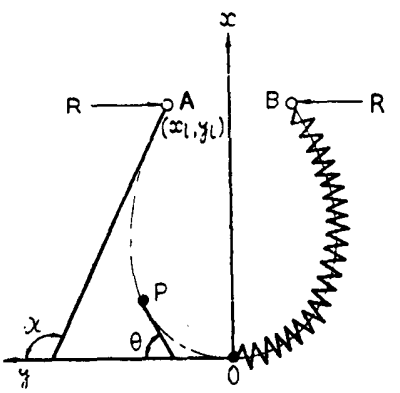

Fig. 1. rical relative to abscissa. As in elastica, the curve of the central line of a coiled spring will be easily determined from the view-point of large deflection problem by the cyordinates of any point $P$ on the deformed cnrve as long as this is in a plane.

We shall denote as follows:

$\theta:$ angle of the tangent to the deflection curve on any given point $P$ and the direction of load $R, \pi \geq \theta \geq 0$, being $\theta=\alpha$ at $A$ and $\theta=0$ at $O$,

$s$ : length $O P$ along the deflection curve of the central line of the spring,

$s_{0}$ : length $s$ under no load,

$l$ : total length of the spring under load $R$,

$l_{0}$ : total length of the spring under no load,

$h$ : pitch at $P$ under load $R$,

$h_{0}$ : pitch at $P$ under no load,

$E, G, m$ : Young's modulus, modulus of rigidity and Poisson's number of the material of spring respectivly,

$I$ : moment of inertia of the cross sectional area of wire,

$r$ : mean radius of the coil.

For simplicity, let the cross section of wire be circular, then we get the polar moment of inertia of area $J=2 I$

Denoting by $A_{0}, B_{0}, C_{0}$ the rigidities for axial force, flexure and shear of the spring under no load respectivel, we get after Biezeno and Grammel ${ }^{10), 11 \text { ) }}$

8) Suhara: Jourual of the Japan Society of Mechanical Engineers 41. No. 255 (June 1938), 583.

9) Mizuno: Reports of Research of Long Columns, p. 87, (March 1952).

10) C. B. Biezeno u. R. Grammel: Technische Dynamik, 2. Aufl. Bd. I., S. 616, Berlin, 1953.

11) S. Timoshenko: Theory of Elastic Stability, Chap 2, §30, 1936. 


$$
A_{0}=\frac{G I h_{0}}{\pi r^{3}}, \quad B_{0}=\frac{2 E G I h_{0}}{\pi r(E+2 G)}, \quad C_{0}=\frac{E I h_{0}}{\pi r^{3}}
$$

Cases of wire with cross section other than circular, e.g. rectangular, may be treated by the method described in the literature mentioned above ${ }^{10}$.

Now, let $T, Q, M$ denote axial force, shearing force and bending moment respectively at point $P$ on the central line of the spring and let tension be counted positive. Then we get

$$
T=-R \cos \theta, \quad Q=R \sin \theta, \quad M=R \int_{0}^{l / 2} \sin \theta d s
$$

and

$$
\frac{T}{A_{0}}=\frac{d s-d s_{0}}{d s_{0}}=\frac{h-h_{0}}{h_{0}}
$$

so

$$
\frac{h}{h_{0}}=1+\frac{T}{A_{0}}=1-\frac{R}{A_{0}} \cos \theta
$$

Therefore, if we denote by $A, B, C$ the rigidities at point $P$ under load $R$, we get

$$
\begin{aligned}
& A=A_{0} \frac{h}{h_{0}}=A_{0}\left(1-\frac{R}{A_{0}} \cos \theta\right) \\
& B=B_{0} \frac{h}{h_{0}}=B_{0}\left(1-\frac{R}{A_{0}} \cos \theta\right) \\
& C=C_{0} \frac{h}{h_{0}}=C_{0}\left(1-\frac{R}{A_{0}} \cos \theta\right)
\end{aligned}
$$

On the other hand, we find the curvature of deflection curve of the central line of the spring as follows ${ }^{11)}$ :

$$
\frac{d \theta}{d s}=\frac{M}{B}+\frac{1}{C} \frac{d Q}{d s}
$$

This is the fundamental equation of deflection curve. If we put Eqs. $(1 \cdot b)$ and $(1 \cdot d)$ into Eq. $(1 \cdot 1)$, we get

$$
\frac{d \theta}{d s}=\frac{R \int_{8}^{l / 2} \sin \theta d s}{B_{0}\left(1-\frac{R}{A_{0}} \cos \theta\right)}+\frac{R \cos \theta}{C_{0}\left(1-\frac{R}{A_{0}} \cos \theta\right)} \cdot \frac{d \theta}{d s}
$$

In order to make the quantities non-dimensional, We put

$$
\left.\begin{array}{l}
s=l \sigma, \quad \frac{R}{A_{0}}=\lambda, \quad l^{2} \frac{R}{B_{0}}=\mu, \quad \frac{R}{C_{0}}=\nu, \\
\lambda+\nu=R\left(3+\frac{2}{m}\right) \frac{\pi r^{3}}{E I h_{0}}=\tau
\end{array}\right\}
$$

then

$$
(1-\tau \cos \theta) \frac{d \theta}{d s}=\mu \int_{0}^{1 / 2} \sin \theta d \sigma
$$


and

$$
\begin{aligned}
& \frac{d}{d \sigma}\left\{(1-\tau \cos \theta) \frac{d \theta}{d \sigma}\right\}=-\mu \sin \theta \\
& \left\{(1-\tau \cos \theta) \frac{d \theta}{d \sigma}\right\} \frac{d}{d \sigma}\left\{(1-\tau \cos \theta) \frac{d \theta}{d \sigma}\right\} d \sigma=-\mu(1-\tau \cos \theta) \sin \theta d \theta \\
& \left.\quad(1-\tau \cos \theta)^{2}\left(\frac{d \theta}{d \sigma}\right)\right)^{2}=\mu\left(2 \cos \theta-\tau \cos ^{2} \theta+H\right)
\end{aligned}
$$

where $H$ is an integration constant. At point $A$, i. e. at $\theta=\alpha$, we have

$$
\frac{d \theta}{d \sigma}=l \frac{d \theta}{d s}=0
$$

so we get

$$
H=-\left(2 \cos \alpha-\tau \cos ^{2} \alpha\right)=\frac{1}{\tau}\left\{(1-\tau \cos \alpha)^{2}-1\right\}
$$

If we put Eq. $(1 \cdot 4)$ into Eq. $(1 \cdot 3)$,

$$
(1-\tau \cos \theta)^{2}\left(\frac{d \theta}{d \sigma}\right)^{2}=\frac{\mu}{\tau}\left\{(1-\tau \cos \alpha)^{2}-(1-\tau \cos \theta)^{2}\right\}
$$

Noting Eq. $(1 \cdot e)$ and putting

$$
\sqrt{\frac{\tau}{\mu}}=\frac{1}{l} \sqrt{\frac{1 / A_{0}+1 / C_{0}}{1 / B_{0}}}=\frac{r}{l} \sqrt{\frac{3 m+2}{2 m+1}}=\frac{r}{l} \sqrt{f}
$$

we get

$$
d \sigma=\sqrt{f} \frac{r}{l} \cdot \frac{(1-\tau \cos \theta) d \theta}{\sqrt{(1-\tau \cos \alpha)^{2}-(1-\tau \cos \theta)^{2}}}
$$

where we assumed $1 \geq \tau$ for the sake of simplicity and took note that

$$
1-\tau \cos \theta \geq 0, \quad 1-\tau \cos \alpha \geq 0 \text { and } d \theta / d \sigma \geq 0
$$

As $d s=l d \sigma$ by Eq. $(1 \cdot \mathrm{e})$, putting

$$
F(\theta)=(1-\tau \cos \alpha)^{2}-(1-\tau \cos \theta)^{2}
$$

we get by Eq. $(\mathbf{1 \cdot 5})$ :

and

$$
d s=\sqrt{f} \cdot \frac{r}{l} \cdot l \cdot \frac{(1-\tau \cos \theta)}{\sqrt{F(\bar{\theta})}} d \theta
$$

$$
\frac{2}{l}=\sqrt{\bar{f}} \cdot r \int_{0}^{\infty} \frac{(1-\tau \cos \theta)}{\sqrt{F(\theta)}} d \theta
$$

The coordinates of point $A$ are as follows:

$$
\begin{aligned}
x_{l} & =\sqrt{f} \cdot r \int_{0}^{\infty} \frac{(1-\tau \cos \theta) \sin \theta}{\sqrt{F(\theta)}} d \theta=\sqrt{f} \cdot r \frac{1}{\tau}|-\sqrt{F(\theta)}|_{0}^{\alpha} \\
& =\sqrt{f} \cdot r \frac{1}{\tau} \sqrt{(1-\tau \cos \alpha)^{2}-(1-\tau)^{2}}
\end{aligned}
$$




$$
y_{l}=\sqrt{ } \bar{f} \cdot r \int_{0}^{\infty} \frac{(1-\tau \cos \theta) \cos \theta}{\sqrt{\overline{F(\theta)}}} d \theta
$$

As $d s / d s_{0}=1-\lambda \cos \theta$ by Eq. (1.c) and $\tau=\lambda+\nu$ by Eq, (1.e), we get

$$
\begin{aligned}
\frac{l_{0}}{2}=\int_{0}^{s} \frac{d s}{1-\lambda \cos \theta} & =\sqrt{f} \cdot r\left[\left(1+\frac{\nu}{\lambda}\right) \int_{0}^{\infty} \frac{d \theta}{\sqrt{\bar{F}(\theta)}}-\frac{\nu}{\lambda} \int_{0}^{\infty} \frac{d \theta}{(1-\lambda \cos \theta) \sqrt{F(\theta)}}\right] \\
& =\sqrt{f} \cdot r \int_{0}^{\infty} \frac{(1-\tau \cos \theta) d \theta}{(1-\lambda \cos \theta) \sqrt{F(\theta)}}
\end{aligned}
$$

The equations (1.A, B, C, D) denote the ihtegrations along $O A$ in Fig. 1 and so express the values at point $A$, but if we take $\theta$ as the upper limit of integration, we get formulae for $s, x, y, s_{0}$ at an arbitrary point $P$ in general:

$$
\begin{aligned}
& s=\sqrt{f} \cdot r \int_{0}^{\theta} \frac{(1-\tau \cos \theta)}{\sqrt{F(\theta)}} d \theta \\
& x=\sqrt{f} \cdot r \frac{1}{\tau} \sqrt{F(\theta)} \\
& y=\sqrt{f} \cdot r \int_{0}^{\theta} \frac{(1-\tau \cos \theta) \cos \theta}{\sqrt{\bar{F}(\theta)}} d \theta \\
& s_{0}=\sqrt{\bar{f}} \cdot r\left[\left(1+\frac{\nu}{\lambda}\right) \int_{0}^{\theta} \sqrt{\frac{d \theta}{F(\theta)}}-\frac{\nu}{\lambda} \int_{0}^{\theta} \frac{d \theta}{(-1 \lambda \cos \theta) \sqrt{F(\theta)}}\right]
\end{aligned}
$$

where

$$
\begin{aligned}
& f=\frac{3 m+2}{2 m+1}, \quad \frac{\nu}{\lambda}=\frac{A_{0}}{C_{0}}=\frac{G}{E}=\frac{m}{2(m+1)} \\
& \tau=R\left(3+\frac{2}{m}\right) \frac{\pi r^{3}}{E I h_{0}} \\
& F(\theta)=(1-\tau \cos \alpha)^{2}-(1-\tau \cos \theta)^{2}
\end{aligned}
$$

$f, \nu / \lambda, \tau$ being constants depending on the dimensions of the spring under no load and elastic modulus of its material. Generally, the integrals on the right of Eqs. $(1 \cdot \mathrm{A}, \mathrm{B}, \mathrm{C}, \mathrm{D})$ are reduced to elliptic integrals as in the case of elastica.*

The fact that the deflection curve of the central line of a buckled coiled spring is expressible in elliptic integrals similar to elastica may be of interest.

N. B. the above calculations refer to the deformation in which the coils of the spring do not touch each other.

* As shown in the appendix of this paper 


\section{Numerical Results and Relations with Usual Data}

Now we shall calculate the numerical values of the solutions obtained in Chap. II. Put Eq. (1.g) into Eq. (1·A), then we get

$$
\frac{l}{2}=l \sqrt{\frac{\tau}{\mu}} \int_{0}^{\alpha} \frac{(1-\tau \cos \theta) d \theta}{\sqrt{(1-\tau \cos \alpha)^{2}-\left(1-\tau \cos \theta^{2}\right)}}
$$

If we transform the variable $\theta$ into $\varphi$ by the definition

$$
\sin ^{2} \frac{\theta}{2}=\sin ^{2} \frac{\alpha}{2} \sin ^{2} \varphi
$$

we have

$$
\begin{gathered}
\varphi=0 \text { at } \theta=0, \varphi=\frac{\pi}{2} \text { at } \theta=\alpha \\
\cos \theta=1-2 \sin ^{2} \frac{\theta}{2}=1-2 \sin ^{2} \frac{\alpha}{2} \sin ^{2} \varphi \\
d \theta=\frac{2 \sin \frac{\alpha}{2} \cos \varphi}{\sqrt{1-\sin ^{2} \frac{\alpha}{2} \sin ^{2} \varphi}} d \varphi \\
\sqrt{(1-\tau \cos \alpha)^{2}-(1-\tau \cos \theta)^{2}}=2 \sqrt{\tau} \sin \frac{\alpha}{2} \cos \varphi \sqrt{1-\tau+\tau\left(1+\sin ^{2} \varphi\right) \sin ^{2} \frac{\alpha}{2}}
\end{gathered}
$$

and

$$
\sqrt{\mu}=2 \int_{0}^{\pi / 2} \frac{\left(1-\tau+2 \tau \sin ^{2} \frac{\alpha}{2} \sin ^{2} \varphi\right) d \varphi}{\sqrt{1-\sin ^{2} \frac{\alpha}{2} \sin ^{2} \varphi} \sqrt{1-\tau+\tau\left(1+\sin ^{2} \varphi\right) \sin ^{2} \frac{\alpha}{2}}}
$$

or expanding into series

$$
\begin{aligned}
\sqrt{\mu} & =2 \int_{0}^{\pi / 2}\left\{(1-\tau)+2 \tau \sin ^{2} \varphi \sin ^{2} \frac{\alpha}{2}\right\}\left(1+\frac{1}{2} \sin ^{2} \varphi \sin ^{2} \frac{\alpha}{2}+\frac{3}{8} \sin ^{4} \varphi \sin ^{4} \frac{\alpha}{2}+\cdots \cdots\right) \\
& \times(1-\tau)^{-1 / 2}\left\{1-\frac{1}{2} \frac{\tau\left(1+\sin ^{2} \varphi\right)}{1-\tau} \sin ^{2} \frac{\alpha}{2}+\frac{3}{8} \frac{\tau^{2}\left(1+\sin ^{2} \varphi\right)^{2}}{(1-\tau)^{2}} \sin ^{4} \frac{\alpha}{2}-\cdots \cdots\right\} d \varphi \\
& =2(1-\tau)^{-1 / 2} \int_{0}^{\pi / 2}\left[(-\tau)+\frac{1}{2}\left\{-\tau+(1+2 \tau) \sin ^{2} \varphi\right\} \sin ^{2} \frac{\alpha}{2}\right. \\
& \left.+\frac{1}{8}\left\{(3+5 \tau) \sin ^{4} \varphi+\frac{3 \tau^{2}-2 \tau \sin ^{2} \varphi-\left(2 \tau+3 \tau^{2}\right) \sin ^{4} \varphi}{1-\tau}\right\} \sin ^{4} \frac{\alpha}{2}+\cdots \cdots\right] d \varphi
\end{aligned}
$$

As

$$
\int_{0}^{\pi / 2} \sin ^{2} \varphi d \varphi=\frac{1}{2} \cdot \frac{\pi}{2}, \quad \int^{\pi / 2} \sin ^{4} \varphi d \varphi=\frac{3}{8} \cdot \frac{\pi}{2}, \cdots \cdots
$$


we get finally

$\left.\begin{array}{ll} & \sqrt{\mu}=\pi(1-\tau)^{-1 / 2} S_{1}, \\ \text { where } & S_{1}=(1-\tau)+\frac{1}{4} \sin ^{2} \frac{\alpha}{2}+\frac{1}{64} \cdot \frac{9-8 \tau}{1-\tau} \sin ^{4} \frac{\alpha}{2}+\cdots \cdots\end{array}\right\}$

when $\alpha=0$, i.e. under buckling load

$$
S_{1}=1-\tau_{c r}
$$

so we have

$$
\sqrt{\mu_{c r}}=\pi\left(1-\tau_{r r}\right)^{1 / 2}
$$

Taking note that $\tau_{c r}=\lambda_{c r}+\nu_{c r}$ (cf. Eq. (1·e)), we can transform Eq. $(2 \cdot 3)$ as follows:

$$
\frac{1}{\frac{\mu_{c r}}{\pi^{2}}+\nu_{c r}}=\frac{1}{1-\lambda_{c r}}=\frac{2}{\lambda_{c r}}\left(\frac{1}{1-\lambda_{c r}}-1\right)
$$

Again from Eq. $(1 \cdot e)$

$$
\lambda_{c r}=\frac{R_{c r}}{A_{0}}, \quad \mu_{c r}=\frac{R_{c r}}{B_{0}} l_{c r}{ }^{2}, \quad \nu_{c r}=\frac{R_{c r}}{C_{0}}
$$

and as the central line is straight under buck?ing lcad,

$$
l_{c r}=\left(1-\lambda_{c r}\right) l_{0}, \quad \frac{R_{c r}}{A_{0}}=\lambda_{c r}=\frac{l_{0}-l_{c r}}{l_{0}}
$$

Therefore, we get

$$
\frac{1}{\frac{l_{r r}^{2}}{\pi^{2}} \frac{R_{c r}}{B_{0}}+\frac{R_{c r}}{C_{0}}}=\frac{R_{c r}}{A_{0}} \frac{l_{0}-l_{c r}}{l_{0}}
$$

or

$$
R_{c r}=\frac{A_{0}\left(l_{0}-l_{r r}\right)}{l_{0}}=\frac{\pi^{2} B_{0}}{l_{c r} l_{0}} \frac{1}{1+\frac{\pi^{2}}{l_{r r}} \frac{B_{0}}{C_{0}}}
$$

The last formula coincides with that of the ordinary theory of coiled springs under buckling load. ${ }^{12)}$ We must notice that $l_{c r}, B_{c r}, C_{c r}$ are the total length, flexual and shearing rigidities under a load exactly equal to $R_{c r}$ and depending on $R_{c r}$. So $R_{c r}$ cannot be determined directly by Eq, $(2 \cdot 4)$ and its calculation requires the solution of an equation of third order given below Eq. $(2 \cdot \mathrm{f})$.

Again transforming the variable in Eq. (1.D) by Eq. (2.a) we get, just as before,

$$
\frac{l_{0}}{2}=\frac{l}{\sqrt{\mu}} \int_{0}^{\pi / 2} \frac{d \varphi}{1-\lambda+2 \lambda \sin ^{2} \varphi \sin ^{2} \frac{\alpha}{2}} \cdot \frac{1-\tau+2 \tau \sin ^{2} \varphi \sin ^{2} \frac{\alpha}{2}}{\sqrt{1-\sin ^{2} \varphi \sin ^{2} \frac{\alpha}{2}} \sqrt{1-\tau+\tau\left(1+\sin ^{2} \varphi\right) \sin ^{2} \frac{\alpha}{2}}}
$$

or, expanding and taking note of the calculation in Eq. $(2 \cdot 2)$, we have

12) S. Timoshenko: Ibid. p. 165, formula (c) etc.. 


$$
\begin{aligned}
\frac{l_{0}}{l} & =\frac{2}{\sqrt{\mu}}(1-\lambda)^{-1}(1-\tau)^{-1 / 2} \int_{0}^{\pi / 2}\left\{1-\frac{2 \lambda}{1-\lambda} \sin ^{2} \varphi \sin ^{2} \frac{\alpha}{2}+\frac{4 \lambda^{2}}{(1-\lambda)^{2}} \sin ^{4} \varphi \sin ^{4} \frac{\alpha}{2}-\cdots \cdots\right\} \\
& +\left[(1-\tau)+\frac{1}{2}\left\{-\tau+(1+2 \tau) \sin ^{2} \varphi\right\} \sin ^{2} \frac{\alpha}{2}+\cdots \cdots\right] d \varphi
\end{aligned}
$$

or

$$
\frac{l_{0}}{l}=(1-\lambda)^{-1}\left[1+\frac{1}{S_{1}}\left\{-\frac{1-\tau}{1-\lambda} \lambda \sin ^{2} \frac{\alpha}{2}-\frac{\lambda}{8} \frac{(3-15 \lambda+2 \tau+10 \lambda \tau)}{(1-\lambda)^{2}} \sin ^{4} \frac{\alpha}{2}-\cdots \cdots\right\}\right]
$$

or, finally,

where

$$
\left.\begin{array}{l}
\frac{l_{0}}{l}=(1-\lambda)^{-1}\left[1-\frac{S_{2}}{S_{1}}\right], \\
S_{2}=\lambda \frac{1-\tau}{1-\lambda} \sin ^{2} \frac{\alpha}{2}+\frac{\lambda}{8} \frac{(3-15 \lambda+2 \tau+10 \lambda \tau)}{(1-\lambda)^{2}} \sin ^{4} \frac{\alpha}{2}+\cdots \cdots
\end{array}\right\}
$$

When $\alpha=0$, i. e. before buckling, we have $S_{2}=0$, therefore

$$
\frac{l_{0}}{l}=(-\lambda)^{1}
$$

This formula contains Eq. $(2 \cdot \mathrm{c})$ in it. Denote $\delta_{y}$, the deflection of the spring before buckling, by $n=l_{0} / h_{0}$, number of turns, and by $d$, diameter of wire, then

or

$$
\begin{aligned}
& \delta_{y}=l_{0}-l=\lambda l_{0}=\frac{R}{A_{0}} l_{0}=\frac{\pi r^{3} l_{0}}{G I h_{0}} R \\
& \delta_{y}=\frac{64 n r^{3}}{d^{4} G} R
\end{aligned}
$$

which coincides with the ordinary formula of coiled springs.

Now, in order to compute the load and deformation after buckling, we must, at first, determine the relation between $\tau$ and $\sin \alpha / 2$.

Noting Eq. (1.g), we get

$$
\frac{\sqrt{\mu}}{l}=\frac{1}{r} \frac{\sqrt{\tau}}{\sqrt{f}}
$$

where $f=\frac{3 m+2}{2 m+1}, m$ being Poisson's number.

In the following we take $m=\frac{10}{3}$, then $\sqrt{f}=1.25$

From Eq. $(2 \cdot 2)$ and $(2 \cdot 5)$, we get

$$
\sqrt{\mu} \frac{l_{0}}{l}=\pi(1-\lambda)^{-1}(1-\tau)^{-1 / 2}\left[S_{1}-S_{2}\right]
$$

or

$$
\frac{1}{\pi \sqrt{f}} \frac{l_{0}}{r} \sqrt{\tau(1-\tau)}(1-\lambda)=S_{1}-S_{2}
$$

when $m=\frac{10}{3}$, from Eq. (1·e) we get 


$$
\frac{\lambda}{\tau}=\frac{\lambda}{\lambda+\nu}=\frac{1}{1+\frac{G}{E}}=\frac{3 m+2}{2 m+2}=0.722
$$

So the above formula may be expressed as follows:

$$
a_{0}=a_{1} \sin ^{2} \frac{\alpha}{2}+a_{2} \sin ^{4} \frac{\alpha}{2}+\cdots \cdots
$$

If we may neglect the terms of $\sin ^{6} \alpha / 2$ and higher powers, we have

where

$$
\sin ^{2} \frac{\alpha}{2}=\frac{-a_{1}+\sqrt{a_{1}^{2}+4 a_{0} a_{1}},}{2 \dot{a}_{2}},
$$

$$
\left.\begin{array}{l}
a_{0}=\frac{1}{\pi} \frac{1}{1.25} \frac{l_{0}}{r} \sqrt{\tau(1-\tau)}(1-0.722 \tau)-(1-\tau) \\
a_{1}=\frac{1}{4}-0.722 \tau \frac{1-\tau}{1-0.722 \tau} \\
a_{2}=\frac{1}{64} \frac{9-8 \tau}{1-\tau}-\frac{0.722}{8} \tau \frac{3-8.33 \tau+7.22 \tau^{2}}{(1-0.722 \tau)^{2}}
\end{array}\right\}
$$

This formula expresses the dependence of $\sin \alpha / 2$ on $\tau$. When $a_{0}=0, \sin \alpha / 2=0$, and this corresponds to buckling load, we have

$$
\frac{\pi^{2} r^{2}}{l_{0}^{2}} f=\frac{\tau_{c r}}{1-\tau_{c r}}\left(1-\lambda_{c r}\right)^{2}
$$

If we put

$$
M=\frac{\pi^{2} r^{2}}{l_{0}^{2}} \frac{1}{1+\frac{2}{m}}, \quad\left(1-\lambda_{c r}\right)=w
$$

and note Eq. $(2 \cdot d)$ and $(2 \cdot e)$, we get

$$
\frac{\pi^{2} r^{2}}{l_{0}^{2}} f=M \frac{3 m+2}{m}
$$

and

$$
\frac{\tau_{c r}}{1-\tau_{c r}}\left(1-\lambda_{c r}\right)^{2}=\frac{(3 m+2) \lambda_{c r}}{2 m+2-(3 m+2) \lambda_{c r}}\left(1-\lambda_{c r}\right)^{2}=\frac{(1-w) w^{2}}{-1+(3+2 / m) w} \frac{3 m+2}{m}
$$

Then

$$
M=\frac{(1-w) w^{2}}{-1+(3+2 / m) w}
$$

or

$$
w^{3}-w^{2}+(3+2 / m) M w-M=0
$$

which coincides with the current formula for the coiled spring under buckling load. ${ }^{132}$

By Eq. $(2 \cdot \mathrm{f})$, we can determine the value $\tau_{c r}$ for each value of $l_{0} / r$.

On the other hand, if we put

$$
K=\frac{R}{R_{c r}}=\frac{\tau}{\tau_{c r}}
$$

13) S. Timoshenko: Ibid. p. 169, formula (i) etc.. 
and regard $K$ as a parameter, we obtain Fig. 2 which shows the dependence of $\tau$ on $l_{0} / r$.

Noting this dependence, the value of $\sin \alpha / 2$ for each $l_{0} / r$ and $K$ is determined from the equation $(2 \cdot 8)$. And we obtain Fig. 3.

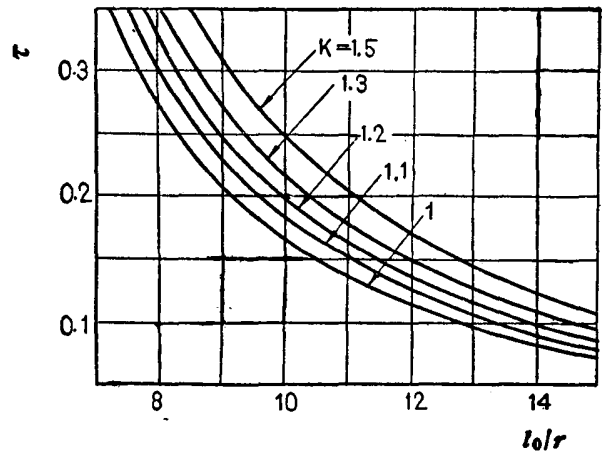

Fig. 2.

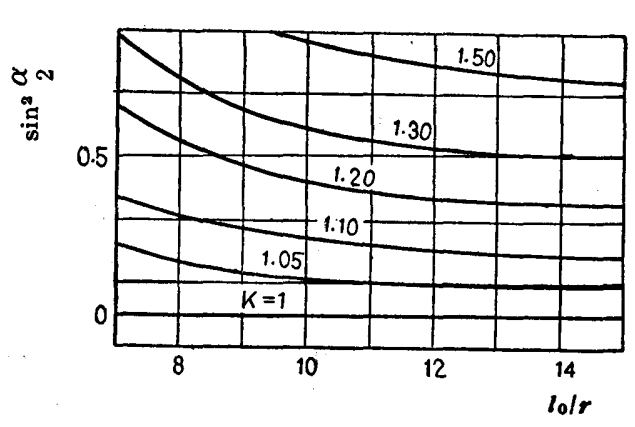

Fig. 3.

The case in which $K=1$ (Fig. 2) corresponds exactly to the Euler's curve of columus. Ordinarily, $\lambda$ is taken as ordinate. ${ }^{14)}$ In the limit $l_{0} / r \rightarrow 0, \tau=1$ independently of $m$. but $\lambda$ depends on $m$. The reason why the abscissa is limited in the figure of this paper is that in the only case of practical importance for buckling holds $l_{0} / r \geq 8$ and when $l_{0} / r>15$, the curve coincides nearly with the horizontal asymptote.

Here we cite Eq. $(1 \cdot \mathrm{B})$ :

$$
x_{l}=\sqrt{f r}\left[\frac{1}{\tau} \sqrt{(1-\tau \cos \alpha)^{2}-(1-\tau)^{2}}\right]
$$

or

$$
\frac{x_{l}}{l_{0}}=2 \sqrt{ } \bar{f} \frac{r}{l_{0}} \frac{\sin \alpha / 2}{\sqrt{ } \tau} \sqrt{1-\tau+\tau \sin ^{2} \frac{\alpha}{2}}
$$

If we replace the variable in Eq. $(1 \cdot \mathrm{C})$ by $\varphi$ following Eq. $(2 \cdot \mathrm{a})$, we get

$$
y_{l}=\frac{l}{\sqrt{\mu}} \int_{0}^{\pi / 2}\left(1-2 \sin ^{2} \varphi \sin ^{2} \frac{\alpha}{2}\right)\left[(1-\tau)+\frac{1}{2}\left\{-\tau+(1+2 \tau) \sin ^{2} \varphi\right\} \sin ^{2} \frac{\alpha}{2}+\cdots \cdots\right] d \varphi
$$

or

$$
2 y_{l}=l\left[1-\frac{1}{S_{1}}\left\{(1-\tau) \sin ^{2} \frac{\alpha}{2}+\frac{1}{8}(3+2 \tau) \sin ^{4} \frac{\alpha}{2}+\cdots \cdots\right\}\right]
$$

and finally

where

$$
\left.\begin{array}{l}
\frac{2 y_{l}}{l_{0}}=(1-\lambda) \frac{S_{1}-S_{3}}{S_{1}-S_{2}}, \\
S_{3}=(1-\tau) \sin ^{2} \frac{\alpha}{2}+\frac{1}{8}(3+2 \tau) \sin ^{4} \frac{\alpha}{2}+\cdots \cdots
\end{array}\right\}
$$

14) S. Timoshenko: Ibid. fig. 103. 
Figure 4 shows the relations of Eqs. $(2 \cdot 9)$ and $(2 \cdot 10)$, in which the results of Figs. 2 and 3 being considered. In it, $x_{l} / l_{0}$ are represented by the family of curves which intersect the left ordinate at $K=1$. The central line of the Spring for $K \leq 1$ is a straight line; $x_{l}=0,2 y_{l} / l_{0}$ are represented by the family of curves which intersect the right ordinate at $K=0$. As

$$
2 y_{l}+\delta_{y}=l_{0}
$$

we can measure the abscissa of this family of curves by $\delta_{y} / l_{0}$, then its origin will be at the right corner as is shown in the bracket ( ) under the abscissa. Now the quantity in case $K \leq 1$ is determined by the usual formula of deflection and is proportional to load. Then we have a straight line as shown in the figure. For comparison, a curve corresponding to elastica is shown in chain lines and the one by the theory of stability in broken linds.

In the curve corresponding to elastica,

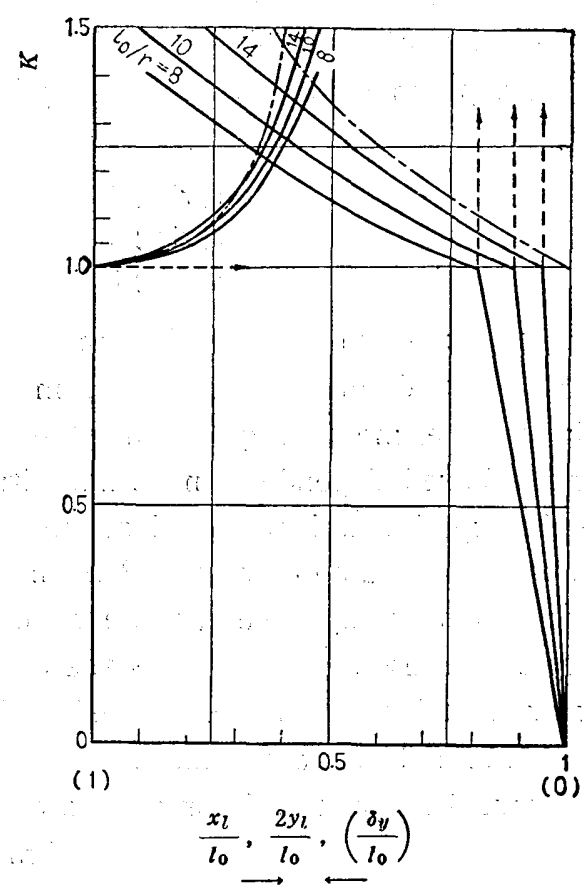

Fig. 4. $x_{l} / l_{0}$ tends to the vertical axis passing through origin 0 and $y_{l} / l_{0}$ to the vertical line through -1 . But in the curves for the springs only $x_{l} / l_{0}$ tends to the vertical axis through the origin, but $y_{l} / l_{0}$ may attain $-\infty$ theoretically as the central line extends indefinitely. Notwithstanding, the intersection of the curve and the vertical axis through origin means the cnincidence of both ends of the spring, and the coils must touch each other. Then we know that the range shown in Fig. 4 is sufficient for practical purposes.

\section{Comparison of the Calculated Results of Deflection after Buckling with the Experiments}

\section{[a] Method of Experiments}

(1) Test Machine Used

We used a universal $5 t$ Olsen testing machine whose loading system is of screw type, not of oil pressure type as in Amsler machine. It allows convenient measurements of deformation of springs which are attached to two tables movable in parallel and may be fixed at any desired pressed position.

But the capacity of our machine was too large compared to the load applied to the springs to measure it accurately. So we were obliged to put aside the measurement of the loads. (As may be seen in the following, their measurement means 
little, as the measurements of deformation alone permit accurate comparison between the measured and the theoretical values.)

(2) Dimensions of Tested Springs

We experimented on 3 kinds of cylindrical coiled springs :

$\begin{array}{ccc} & D \mathrm{~mm} & d \mathrm{~mm} \\ \text { spring } 1 & 25.9 & 2.1 \\ \text { spring 2 } & 24.65 & 4.0 \\ \text { spring 3 } & 20.4 & 5.0\end{array}$

where $D$ : diameter of coil in $\mathrm{mm}$,

$d$ : diameter of wire in $\mathrm{mm}$.

These experiments require only the constancy of the pitch of the coils under no load and are independent on its magnitude. The length of the springs was adapted to the experimental method described in (3) Method of Measurement. As the properties of materials other than Poisson's ratio are known not to affect the measured results of this experiment, these were not investigated in detail. We ascertained only that these springs were made of ordinary steel wire. In the following, the experimental results are compared with the theoretical data in which $m=10 / 3$.

(3) Method of Measurement

Figure 5 shows the deformations of buckled coiled springs. In this figure the

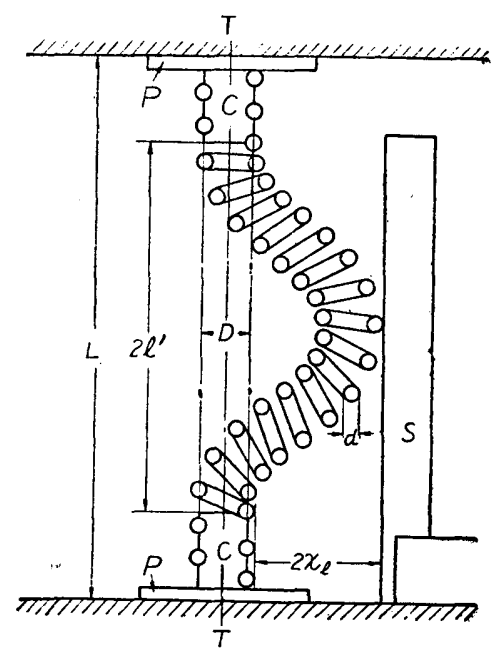

Fig. 5. dimensions which are almost invariable and coincide practically with those under no loads shall be denoted by suffices 0 . That is, $D \subsetneq D_{0}$, $d \fallingdotseq d_{0}$. Again the denotations in III are maintained :

and we have

$$
l^{\prime}=2 y_{l}, \quad l_{0}^{\prime}=l_{0}, \quad l_{0}-l^{\prime}=\delta_{y}
$$

$$
x_{l, 0}=0
$$

But the errors in $x_{l, 0}$ originating in the manufacture of the springs are of the order of $1 / 10$ $\mathrm{mm}$ and cause discrepancies between theory and experiment near the buckling point, as described below.

$C$ in the Fig. 5 is a metallic part fixing the tow ends of spring in exact direction of its central line, and on its cylindrical surface is cut a spiral groove into which spring may be screwed. $P$ is a plate to protect against the displacement of $C$ when springs are buckled.

The experiment was performed in the following order. We fixed the free length $2 l_{0}$ of springs under no load is fixed at $2 l_{0} / D=l_{0} / r=14,10,8$ respectively, these values being used for calculation in III. and we cut the springs with margins to enable their two ends to be screwed in and to be fixed by the metal part $C$. Then we 
attached springs to part $C$ and made the horizontal free length to planned $2 l_{0}$ by adjusting the screwing into $C$ and fixed it on $P$. This was attached between the tables $T$ of the testing machine as in Fig. 5 and adjusted to stand perpendicular to their two planes by a right-angled square $S$.

As we shorten the distance between the two $T$ 's, by driving the testing machine, the spring will be buckled at a certain position. We stop the machine at certain proper positions before and after the buckling point and measure the dimensions $L$ and $2 x_{\imath}$ respectively. We repeat this measurement several times as far as the coils of spring do not touch each other, than unload the spring and ascertain if it retain the original $x_{l, 0}$.

(4) Kinds of Experiments

We have performed various experiments as shown in Table 1.

Table 1.

\begin{tabular}{|l|c|c|c|}
\hline & spring 1 & spring 2 & spring 3 \\
\hline $2 l_{0} / D=l_{0} / r$ & 10,8 & 10,8 & 10,14 \\
\hline
\end{tabular}

\section{[b] Results of Experiments}

(1) Method of Treatment of Results

We show in Fig. 6 a curve expressing the relations between $x_{l} / l_{0}$ and $\delta_{y} / l_{0}$ which is obtained from Fig. 4 calculated in III. In Fig. 6 we plot the measured values $2 \delta_{y}$ and $2 x_{l}$ divided by $2 l_{0}, 2 l^{\prime}$ being the measured $L$ minus the height of $C$ and thickness of $P$. The coincidence of these two will ascertain our theory and especially will prove the accuracy of approximate calculation in III. The range shown in Fig. 6 is more limited than in Fig. 4. This was obliged by the fact that the touching of coils with each other in the experiment does not allow a comparison with the calculation.

(2) Discussion of the Results

The causes of the discrepancy between the experimental plot and the theoretical curve near buckling may be attributed, on one side, to the errors in dimensions of springs, such as $x_{l, 0} \neq 0$ described above and others, and, on the other side, to the incompletely fixed conditions of support which consists particularly in the asymmetric arrangement of springs relative to the central measuring point of springs caused by the

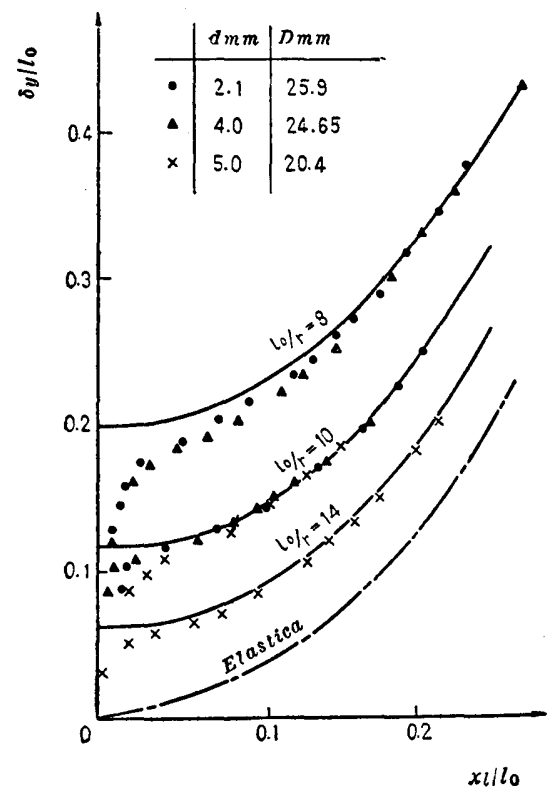

Fig. 6. accurate adjustment of free length. Both are inevitable in our experiments and may occur in any practical springs. Except for this non-coincidence, the experimental points nicely fit the theoretical curve. 


\section{Applications to the Coiled Springs for Precision Mechanism}

In order to limit their bulk as for as possible in precision mechanisms there are often used extension springs whose characteristics are non-linearized by initial tension as shown in Fig. 7. The spring shown in this figure is the one giving the

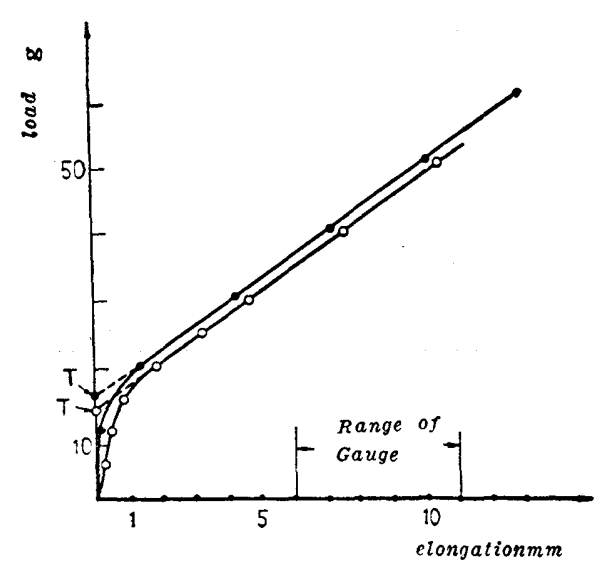

Fig. 7. measuring pressure in dial gauges described below. The real lines show the respective characteristics of the springs attached to two different gauges of the same type and the points show measured values. The intersections $T$ and $T^{\prime}$ with ordinate of the elongated dotted straight line at the end part of these plots show the maghitudes of initial tensions of the two springs each. Distinct difference between $T$ and $T^{r}$ observed in the figure proves the frequent occurrence of errors in initial tension during manufacturing, these

errors often causing variation in performance of springs.

Non-linear characteristics may be obtained in compression spring after buckling as shown in Fig. 4. We have made a compression spring for measuring pressure in dial gauge and compared it with an extension spring. The buckling point in Fig. 4 is not so dependent on the manufacturing conditions as initial tension and is theoretically fixed, only dependent upon elastic coefficients of materials. In the following is described the measuring pressure in the usual dial gauge using an extension spring and both design-computation and experimentally determined measuring pressure when in dial gauge the deformation of buckled compression spring is used.

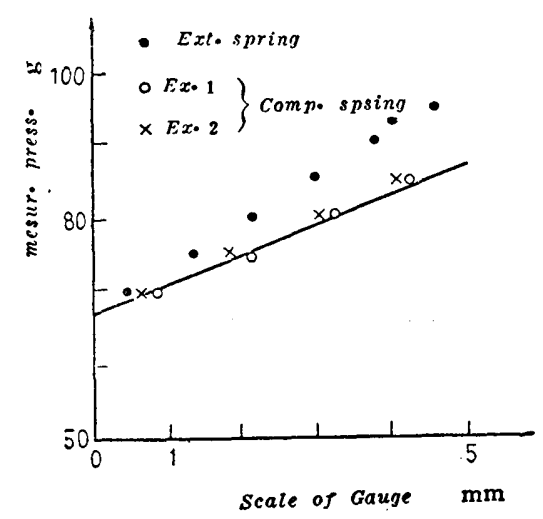

Fig. 8.
The measuring pressure of a dial gauge of usual construction is given by the extension coiled spring working upon spindle and the spiral spring to exclude the play between toothed wheels. We have measured this measuring pressure with the spring testing machine (smallest scale $2 \mathrm{~g}, 1 / 100 \mathrm{~mm}$ ) manufactured by Tokyo Kôki Ltd., and plotted the results in Fig. 8. This gauge has a smallest scale $0.01 \mathrm{~mm}$, a range $5 \mathrm{~mm}$ and is of a usual type. The measured points in this figure correspond to the measurements of spring alone in Fig. 7 which are als: made 
with the same machine. Fig. 7 shows that in the measuring range of the dial gauge using an extension spring the variation in load depends linearly upon elongation. As the allowable elongation is $6 \sim 11 \mathrm{~mm}$, the load in the range $35 \sim 52 \mathrm{~g}$ may be used as measuring pressure. Between this value and that in Fig. 8 there is a difference of $32 \sim 45 \mathrm{~g}$, which is given by the spiral spring.

\section{[a] Calculation of Coiled Compression Spring}

Fig. 4 shows that when $l_{0} / r=14$, the characteristics are almost straight up to $\delta / l_{0}=0.5$ after buckling. Experiments (Fig. 6) show some deviations from the theoretical values near the buckling point but beyond $\delta / l_{0}=0.2$ good agreament with them. Therefore we used the range $\delta / l_{0}=0.2 \sim 0.5$ in the following examples.

Example 1

Diameter of wire $d: 0.35 \mathrm{~mm}$, maen diameter of coil $2 r: 3 \mathrm{~mm}$, pitch $p: 1 \mathrm{~mm}$, total length $l_{0}: 21 \mathrm{~mm}$ and number of turns $n: 21$.

Both ends are hinged and we have

$$
\frac{l_{0}}{r}=14
$$

Then at the buckling point in Fig. 4 (from Eq. (2.f))

$$
\frac{\delta_{\text {cr }}}{l_{0}}=0.059
$$

Therefore the bucling load is:

$$
R_{\mathrm{c} r}=\frac{d^{4} G \delta_{c r}}{64 n r^{3}}=32.5 \mathrm{~g}
$$

From

$$
\frac{\text { Rarge of Gauge }}{\text { Total Length of Spring }}=0.25
$$

and using $\delta / l_{0}$ of $0.24 \sim 0.48$, we get as loads

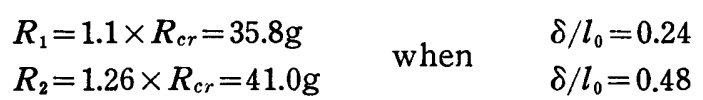

and the distances beteween two ends corresponding to $R_{1}$ and $R_{2}$ become as follows.

$$
\begin{aligned}
& l_{1}=(1-0.24) l_{0}=16 \mathrm{~mm} \\
& l_{2}=(1-0.48) l_{0}=11 \mathrm{~mm}
\end{aligned}
$$

Then the dependence of deflection upon load is nearly linear in this range, as may be seen from the above. Thus we get the calculated values at each end of the measurable range by adding loads $32,45 \mathrm{~g}$ of the above spring respectively to $R_{1}$ r $\boldsymbol{R}_{\mathbf{2}}$. The straight line through these two values shows the calculated measuring pressure and is represented by the real line in Fig. 8. Comparing this with the results obtained by the use of usual extension spring, we see little difference in minimum messuring pressure and less variation in measuring pressure. The total 
free length of the extension spring is $17 \mathrm{~mm}$ and when added with the maximum elongation $11 \mathrm{~mm}$, it will be $28 \mathrm{~mm}$ in its maximal length. In contrast, the maximum total length of compression spring is only $16 \mathrm{~mm}$.

\section{Example 2}

If the diameter of wire of extention springs is changed, its initial tension will necessarily change, but in compression springs we can choose such a pitch that the buckling load may not be affected by the change of wire-diameter. In example 2 we choose proper wire-diameter and pitch (so number of turns, tco) unlike those in Ex. 1 so that the constancy of buckling load may result.

We take

wire-diameter $d: 0.3 \mathrm{~mm}$,

pitch $p: 1.86 \mathrm{~mm}$,

number of turns $n$ : 11.3 .

and other dimensions the same as in Ex. 1. Then we have

$$
R_{c r}=32.5 \mathrm{~g}
$$

and we get same measuring pressure as in Ex. 1 .

Photograph (a) shows the gauge using a usual extension spring, and Photograph (b) the one using the buckled compression spring in Ex. 2. The spring in (a) may elongate by $5 \mathrm{~mm}$ further and the one in (b) may be shortened by $5 \mathrm{~mm}$ still.

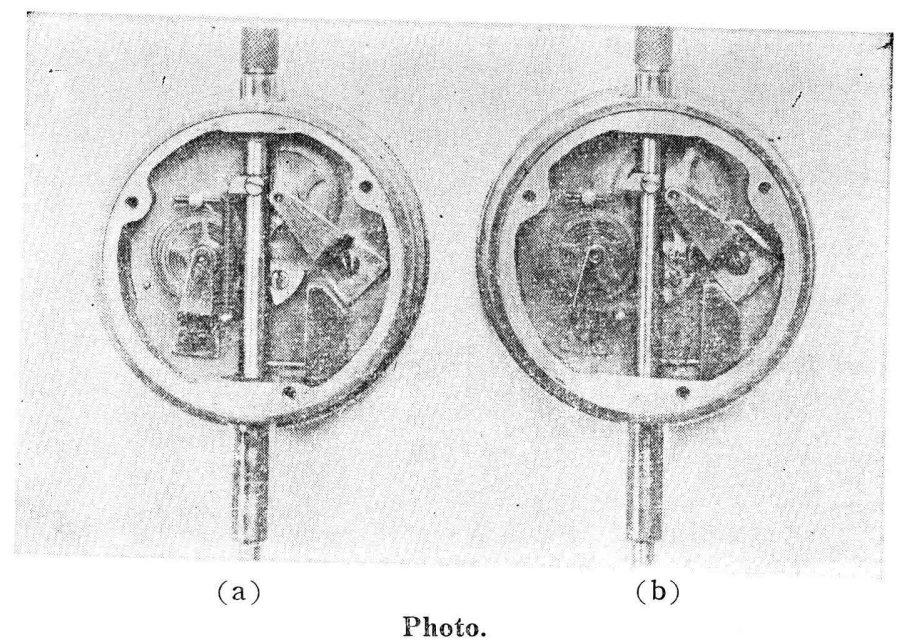

\section{[b] Experiments on Measuring Pressure}

We obtained springs shown in the examples of [a] and attached them in turn to the dial gauge. The measuring pressure determined by the spring test machine is shown in Fig. 8. Thus it is seen that the measured values fit good with the calculated results in [a] and the application of deformation after buckling of compression spring enables the dial gauge to perform much better than the use of usual extension spring. 


\section{Acknowiedgement}

This work was made possible by the support of the Fund for the Promotion of Science in Keio University for 1957. We express our cordial thanks.

\section{Appendix}

Put $\cos \theta=z$ into the integlals on the right of Eqs. (1.A, B, C, D), noting $d \theta=d z /\left(-\sqrt{1-z^{2}}\right)$ we have

$$
\begin{aligned}
& I_{0}=\int_{0}^{\theta} \frac{d \theta}{\sqrt{\overline{F(\theta)}}}=-\int_{1}^{z} \frac{d z}{\tau \sqrt{G(z)}} \\
& I_{1}=\int_{0}^{\theta} \cos \theta \frac{d \theta}{\sqrt{\overline{F(\theta)}}}=-\int_{1}^{z} z \frac{d z}{\tau \sqrt{\overline{G(z)}}} \\
& I_{2}=\int_{0}^{\theta} \cos ^{2} \theta \frac{d \theta}{\sqrt{F(\theta)}}=-\int_{1}^{z} z^{2} \frac{d z}{\tau \sqrt{\overline{G(z)}}} \\
& J=\int_{0}^{\theta} \frac{d \theta}{(1-\lambda \cos \theta) \sqrt{\overline{F(\theta)}}}=\int_{1}^{z} \frac{d z}{\lambda \tau(z-1 / \lambda) \sqrt{G(z)}}
\end{aligned}
$$

where

$$
\begin{aligned}
& F(\theta)=(1-\tau \cos \alpha)^{2}-(1-\tau \cos \theta)^{2} \\
& G(z)=(z-1)(z+1)(z-\cos \alpha)(z+\cos \alpha-2 / \tau)
\end{aligned}
$$

Then, we get after W. Gröbner u. N. Hofreiter: Integraltafel 2 teil, 1958, S. 47, noting $1>\cos \alpha>-1, \infty>2 / \tau-\cos \alpha>1 ; 0<\tau \leq 1$, for example

$$
\begin{aligned}
& I_{\theta=\alpha}=\int_{\cos \alpha}^{1} \frac{d z}{\tau \sqrt{G(z)}}=\frac{1}{\tau \sqrt{1 / \tau-\cos \alpha}} K(k) \\
& I_{\theta=\alpha}=\int_{\cos \alpha}^{1} z \frac{d z}{\tau \sqrt{G(z)}}=\frac{1}{\tau \sqrt{1 / \tau-\cos \alpha}}\left[(1+\cos \alpha) \Pi\left(\frac{\cos \alpha-1}{2}, k\right)-K(k)\right]
\end{aligned}
$$

where

$$
k^{2}=\frac{(2 / \tau-\cos \alpha+1)(1-\cos \alpha)}{4(1 / \tau-\cos \alpha)}
$$

